Table 3. ${ }^{1} \mathrm{H}$ NMR data for TAPA, TIPA and TU in $\mathrm{CDCl}_{3}[\mathrm{a}]$

\begin{tabular}{llllllll}
\hline & $\mathrm{H}_{2}$ & $\mathrm{H}_{3}$ & $\mathrm{H}_{5}$ & $\mathrm{H}_{7}$ & $\mathrm{H}_{8}$ & $\mathrm{H}_{9}$ & $\mathrm{H}_{10}$ \\
\hline TAPA & 6.65 & 6.65 & 3.5 & & & & \\
TIPA & 6.97 & 6.97 & & & & & \\
TU & 7.1 & 7.1 & 6.8 & 4.2 & 1.5 & 1.5 & 0.9
\end{tabular}

[a] Numbering of protons and carbons is indicated in Figure 1.

under dry $\mathrm{N}_{2}$. Tris ( $p$-isocyanatophenyl)amine was obtained in $63 \%$ yield, purified by recrystallization in diethyl ether and has a melting point of $148^{\circ} \mathrm{C}$ $\mathrm{C}_{21} \mathrm{H}_{12} \mathrm{~N}_{4} \mathrm{O}_{3}$ : calc. $\mathrm{C} 68.48 \% \mathrm{H} 3.28 \% \mathrm{~N} 15.21 \%$; found $\mathrm{C} 68.43 \% \mathrm{H} 3.28 \%$ $\mathrm{N} 15.13 \%$. In the IR spectra a very intense peak at $2270 \mathrm{~cm}^{-1}$ due to the isocyanate group is observed. The ${ }^{1} \mathrm{H}$ NMR and ${ }^{13} \mathrm{C}$ NMR data of TIPA in $\mathrm{CDCl}_{3}$ are reported in Tables 3 and 4 , respectively

Table 4. ${ }^{13} \mathrm{C}$ NMR data for TAPA, TIPA and TU in $\mathrm{CDCl}_{3}[\mathrm{a}]$

\begin{tabular}{llllllllll}
\hline & $\mathrm{C}_{1}$ & $\mathrm{C}_{2}$ & $\mathrm{C}_{3}$ & $\mathrm{C}_{4}$ & $\mathrm{C}_{6}$ & $\mathrm{C}_{7}$ & $\mathrm{C}_{8}$ & $\mathrm{C}_{9}$ & $\mathrm{C}_{10}$ \\
\hline TAPA & 143.1 & 114.8 & 124.1 & 138.8 & & & & & \\
TIPA & 146.8 & 125.7 & 124.8 & 128.2 & 124.5 & & & & \\
TU & 143.6 & 124.3 & 122.0 & 132.7 & 154.0 & 85.1 & 30.9 & 19.0 & 13.7
\end{tabular}

[a] Numbering of protons and carbons is indicated in Figure 1.

TIPA was reacted with 1-butanol in 1,2-dichloroethane at $70^{\circ} \mathrm{C}$ under a $\mathrm{N}_{2}$ atmosphere for 2 days. After the solvent had been removed, the reaction product, TU, was purified by using analytical high-pressure liquid chromatography (HPLC) with THF/hexane (20/80) as eluant. TU was obtained as a solid with a melting point of $167{ }^{\circ} \mathrm{C} .{ }^{1} \mathrm{H}$ NMR and ${ }^{13} \mathrm{C} \mathrm{NMR}$ data of TU in $\mathrm{CDCl}_{3}$ are reported in Tables 3 and 4 respectively. $\mathrm{C}_{33} \mathrm{H}_{42} \mathrm{~N}_{4} \mathrm{O}_{6}$ : calc. $\mathrm{C} 67.09 \% \mathrm{H}$ $7.16 \% \mathrm{~N} 9.48 \%$; found C $66.98 \% \mathrm{H} 7.04 \% \mathrm{~N} 9.41 \%$. In the 1R spectra the $2270 \mathrm{~cm}^{-1}$ band disappeared completely while new bands due to the urethane groups appeared at 1710, 1698, and $1598 \mathrm{~cm}^{-1}$.

UV absorption spectra were recorded with a Perkin Elmer Lambda $6 \mathrm{UV} / \mathrm{vis}$ spectrometer. Corrected fluorescence and excitation spectra were obtained with a SPEX Fluorolog 212. All solutions had an absorbance of approximately 0.1 . Third-order susceptibilities of TIPA and TU in solution were measured using an optical phase conjugated (OPC) interferometer. [5] A Nd: YAG laser (YG-501 Quantel) was used as the excitation source. The intensity of the OPC wave generated by degenerate four-wave mixing in a medium is proportional to the third-order susceptibility of this medium. This macroscopic susceptibility $\chi^{(3)}$ is related to the microscopic second-order hyperpolarizability $\gamma$ of the organic molecules present in the sample cell. Carbon disulfide $\left(\chi_{\mathbb{S}_{2}}^{(3)}=6.8 \times 10^{-13}\right.$ esu $)$ was used as a reference.

Received: Novernber 20, 1992 Final version: January 20, 1993

[1] Ji Kang Yoo. C. S. P. Sung, Macromolecules 1991, 24, 410-414. E. Pyun, R. J. Mathisen, C. S. P. Sung, Macromolecules 1989, 22, 1174. P. Dickinson, C. S. P. Sung, Macromolecules 1992, 25, 3758-3768.

[2] X. Y. W. Yu, C. S. P. Sung, Macromolecules 1990, 23, 390-398.

[3] J. K. Yoo, C. S. P. Sung, ACS Polym. Mater. Sci. Eng. 1989, 60, 429.

[4] C. S. P. Sung, E. Pyun, H.-L. Sun, Macromolecules 1986, 19, 2922.

[5] A. Persoons, B. Van Wonterghem, P. Tackx, Proc. Soc. Photo-Opt. Insirum. Eng. 1991, 1409, 220.

[6] G. T. Boyd, J. Opt. Soc. Am. 1989, B6, 685-692.

[7] M. Chen, L. R. Dalton, Macromolecules 1992, 25, 4032-4035.

[8] M. T. Zhao, M. Samoc, B. P. Singh, P. N. Prasad, J. Phys. Chem. 1989, 93 , $7916-7920$.

[9] D. K. Singer, J. E. Sohn, L. A. King, H. M. Gordon, H. E. Katz, C. W. Dirk, J. Opt. Soc, Am. 1989, B6, 1339.

[10] B. F. Levine, C. G. Bethea, J. Chem. Phys. 1975, 63, 2666.

[11] C. S. Willand, D. J. Williams, Ber. Bunsenges. Phys. Chem. 1987, 91, 1304.

[12] D. J. Williams, Nonlinear Optical Properties of Organic and Polymeric Materials, ACS Symp. 233, Am. Chem. Soc., Washington DC 1983.

[13] R. I. Walter, J. Am. Chem. Soc, 1953, 75, 2771-2772.

\section{Optical Second-Harmonic Effect of Sol-Gel Inorganic-Organic Nanocomposites * *}

\author{
By Lothar Kador, ${ }^{*}$ Reiner Fischer, Dietrich Haarer, \\ Reiner Kasemann, Stefan Brück, Helmut Schmidt, \\ and Heinz Dürr
}

The field of nonlinear optics has been characterized by a remarkable increase of the research activities during the past decade. This is mostly due to promising technical applications which could considerably enhance the potential of optics and optoelectronics, espcially in the field of data processing and data storage. ${ }^{[1]}$ For frequency doubling or modulation of light by an electric field, materials with a non-zero quadratic nonlinear coefficient $\left(\chi^{(2)}\right)$ are required.

For general reasons of symmetry, even-order nonlinear coefficients can only be realized in substances with a noncentrosymmetric structure. Thus, polymeric and other disordered materials must be poled by an external field. Usually, nonlinear-optically active (NLO-active) dye molecules, which are characterized by large delocalized $\pi$ electron systems and which, in addition, carry strong electric dipole moments, are incorporated into inert matrices so that orientation of the molecules is possible in an electric field while the samples are kept above the glass transition temperature (which must be well above room temperature). The goal is to permanently freeze in at least part of the orientational order by lowering the temperature below the glass point $\left(T_{\mathrm{g}}\right)$ while the field is still being applied. ${ }^{[2]}$ However, a general problem in commonly known polymeric systems is that the polarization decays with time to some value which is too small for technical applications.

In order to solve this problem, several methods have been proposed. One way is to use organic polymers and/or dopant molecules that carry more than one functional side group per unit so that the sample can be chemically crosslinked during the poling process. ${ }^{[3-5]}$ Reasonable temporal and thermal stability of the $\chi^{(2)}$ parameter has been achieved with the crosslinking technique. However, a general problem is that raising the temperature of the precursor polymer during poling, which is necessary for an effective orientation of the chromophores, at the same time initiates the crosslinking process. Hence, it is difficult to decouple these two processes. A way to circumvent this problem is to prepare rigid matrices in a sol-gel transition. The sol-gel process allows the synthesis of inorganic-organic composite materials which

[*] Dr. L. Kador, Dr. R. Fischer, Prof. D. Haarer

Physical Institute and Bayreuther Institut für Makromolekülforschung (BIMF), University of Bayreuth

Postfach 101251, W-8580 Bayreuth (FRG)

Dr. R. Kasemann, S. Brück. Prof. H. Schmidt

Institut für Neue Materialien (INM)

Im Stadtwald, Gebäude 43, W-6600 Saarbrïcken (FRG)

Dr. H. Dürr

Institute of Organic Chemistry, Universität des Saarlandes

Im Stađtwald, W-6600 Saarbrücken (FRG)

[**] This work was performed with a financial grant from the "VolkswagenStiftung". The work was also supported by the "Fonds der Cherrischen Industrie". 
can be highly crosslinked, and high $T_{\mathrm{g}}$ values (several hundred degrees Celsius) can be obtained. ${ }^{[6-9]}$

The objective of the investigation reported here was to demonstrate the basic suitability of this class of materials as matrices for $\chi^{(2)}$ chromophores and to show that stable poling in an electric field is possible. As a prerequisite, it was necessary to develop sol-gel materials with a minimized concentration of free charge carriers and to employ suitable methods for incorporating $\chi^{(2)}$ chromophores. As was shown by Livage and co-workers, ${ }^{[10]} \mathrm{NLO}$-active dye molecules can be incorporated in sol-gel materials containing $\equiv \mathrm{Si}-\mathrm{CH}_{3}$ groups and second-order nonlinear susceptibilities can be obtained by poling. In our concept, polymerizable organic components were added since they allow us to carry out controlled photo- or thermal polymerization in the course of poling and open up the possibility of performing further shaping processes such as embossing during photo- or thermally initiated crosslinking or photolithography. ${ }^{[9,11]}$ Hence, our matrix materials can be characterized as inorganic-organic nanocomposites or organically modified ceramics (ORMOCERs). For the $\chi^{(2)}$ measurements, we used the Maker fringe technique. ${ }^{[12]}$

In order to optimize the charge carrier concentration $\left(\mathrm{H}^{\oplus}\right.$, $\mathrm{H}_{2} \mathrm{O}$ ) resulting from the sol-gel synthesis, a detailed investigation of the synthesis mechanism was carried out by following the synthesis process (hydrolysis, condensation, $\mathrm{H}_{2} \mathrm{O}$ content, and silanol concentration) by Karl-Fischer titration, IR, and ${ }^{29} \mathrm{Si}$ NMR spectroscopy. Homogeneous films of low optical loss were synthesized by prehydrolyzing the silane with $0.5 \mathrm{H}_{2} \mathrm{O}$ per alkoxy group and then adding $\mathrm{Zr}(\mathrm{OR})_{4}$ complexed with methacrylic acid, MAA $(\mathrm{Zr}: \mathrm{MAA}=1: 1)$. The $\mathrm{Zr}$ alkoxide/MAA complex consumes the $\mathrm{H}_{2} \mathrm{O}$ present in a latent form as $\equiv \mathrm{SiOH}$ without leading to the formation of "free" $\mathrm{H}_{2} \mathrm{O}$. The catalytic activity of $\mathrm{Zr}$ allows us to run the process at extremely low $\mathrm{H}^{\oplus}$ concentrations necessary for high dielectric strengths. Due to the low charge carrier content, the breakdown voltage of the cast unsolidified films is in the range of $0.7 \times 10^{5}$ to $1.0 \times 10^{5} \mathrm{~V} /$ $\mathrm{cm}$, suitable for corona poling. In the course of the sol-gel transition, the concentration of free ions in the matrix, and hence the conductivity, decreases even further, which can be concluded from a slight increase of the corona voltage (using regulation of the current). However, a quantitative measurement of this effect could not be performed. After addition of the $\chi^{(2)}$ chromophores, the viscous liquids were used in a dipor spin-coating process and films of thicknesses between 0.5 and $6 \mu \mathrm{m}$ were obtained, depending on the volume fraction of the saturated dye solution. The freshly prepared films were poled with a corona current of $5 \mu \mathrm{A}$, corresponding to voltages of 6 to $8 \mathrm{kV}$, with no dielectric breakdown occurring. The sol-gel transition was performed by heating the sample films to $120^{\circ} \mathrm{C}$ for $1 \mathrm{~h}$. In the case of UV solidification, the samples were illuminated with UV light for 30 to $60 \mathrm{~s}$ and then heated to $70^{\circ} \mathrm{C}$ for $1 \mathrm{~h}$. Solid yellow or red colored films were obtained which exhibited no agglomeration of the chromophores, as was proven by vis spec- troscopy. Figure 1 shows a structure model of a solidified three-dimensionally crosslinked ORMOCER matrix with a $\chi^{(2)}$-chromophore bonded to the inorganic backbone.

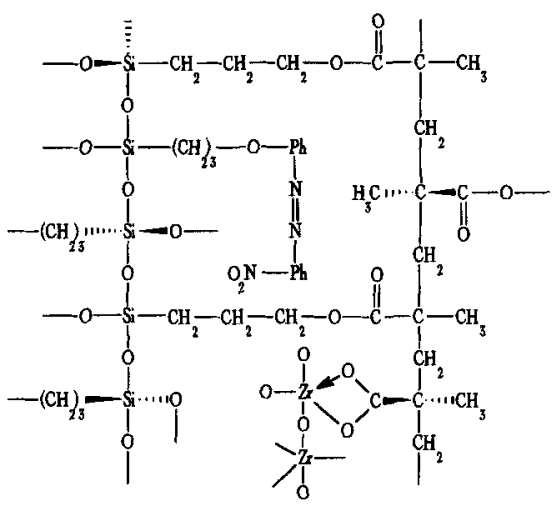

Fig. 1. Structure model of the three-dimensionally crosslinked interconnected inorganic-organic network of a solidified ORMOCER with a $\chi^{(2)}$-chromophore chemically bonded to the inorganic backbone (in the center of the figure).

The results of the Maker fringe measurements are presented in Figures 2-4. Figure 2 a shows a typical second-harmonic signal of a poled sol-gel sample; the irradiated wavelength was $1064 \mathrm{~nm}$ and the sample thickness $2.0 \mu \mathrm{m}$. The NLO-active dye molecules (ANB, 5 wt.- \%; see Scheme) are chemically bonded to the matrix backbone. Since the sample

Scheme, 4-Nitro-4'-(oxypropyltrimethoxysilyl)azobenzene, ANB.

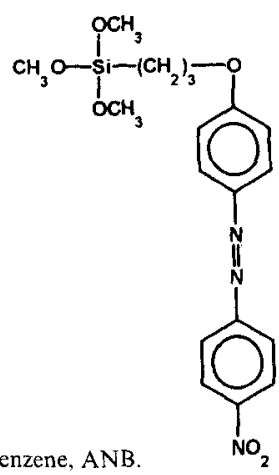

is much thinner than the coherence length, no real Maker fringes appear; only an envelope is visible, which is due to transmission and projection factors. ${ }^{[13]}$ In the case of thin poled films in which the molecules are oriented perpendicularly to the surface, the signal is always zero at normal incidence, since in this geometry the polarization direction of the incident light is perpendicular to the molecular axis of high hyperpolarizability. ${ }^{[14]}$ The dotted line represents the theoretical fit to the data with indices of refraction of $n_{\omega}=1.2104$ 
and $n_{2 \omega}=1.50$ and a $\chi_{33}^{(2)}$ coefficient of $0.02316 \mathrm{pm} / \mathrm{V}$. The fit parameters were $\chi_{33}^{(2)}$ and $n_{\omega}$. The big difference between $n_{\omega}$ and $n_{2 \omega}$ is somewhat unusual and may be due to surface irregularities of the sample film, which affect the signal shape particularly at large angles of incidence. The result for $\chi_{33}^{(2)}$ is influenced by the refractive indices to some extent, but since these are first data of a new class of materials, no effort was made to measure the refractive index values accurately in a separate experiment. For instance, using $n_{\omega}=1.40$ and $n_{2 \omega}=1.50$ yields $\chi_{33}^{(2)}=0.03232 \mathrm{pm} / \mathrm{V}$, which is of the same order of magnitude.
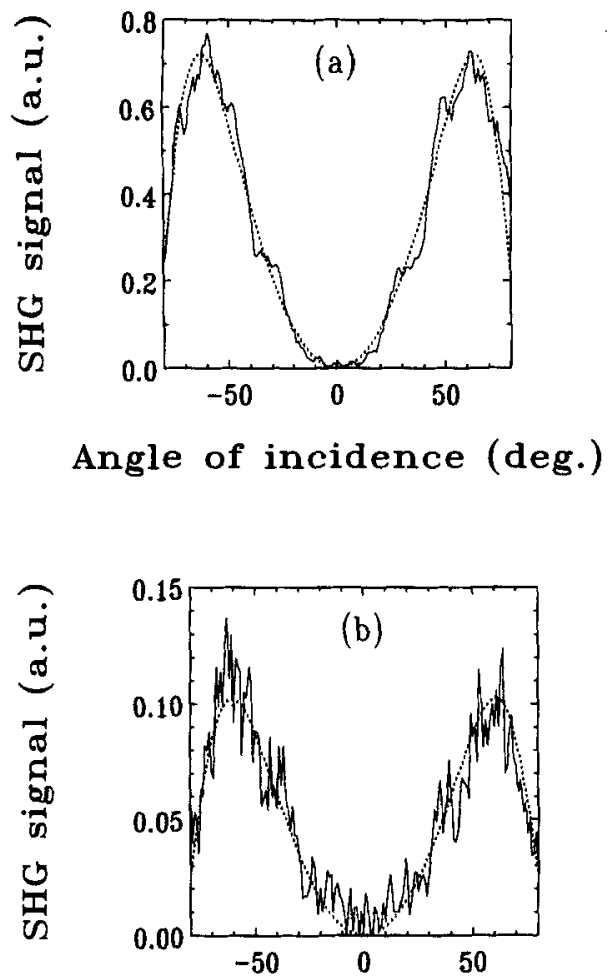

Angle of incidence (deg.)

Fig. 2. Maker fringe signals of a side group ORMOCER sample (containing the dye ANB) at two different irradiated infrared wavelengths: a) $1064 \mathrm{~nm}$, b) $954 \mathrm{~nm}$. The solid lines are the experimental data; the dotted lines represent theoretical fits with nonlinear susceptibilities $\chi_{33}^{(2)}$ of $0.02316 \mathrm{pm} / \mathrm{V}$ (a) and $0.04200 \mathrm{pm} / \mathrm{V}(\mathrm{b})$. In each part the fit parameters were $\chi_{33}^{(2)}$ and the refractive index at the irradiated wavelength, $n_{t p}$. Sample thickness: $2.0 \mu \mathrm{m}$.

In order to check whether the nonlinear susceptibility is affected by resonance enhancement due to absorption of the second harmonic, we performed the Maker fringe experiment at a second infrared wavelength of $954 \mathrm{~nm}$. The signal curve is plotted in Figure $2 \mathrm{~b}$ together with the corresponding fit. The parameters are $\chi_{33}^{(2)}=0.04200 \mathrm{pm} / \mathrm{V}, n_{\omega}=1.4569$, $n_{2 \omega}=1.55$. At $954 \mathrm{~nm}$, the $\chi^{(2)}$ parameter value is larger than at $1064 \mathrm{~nm}$. This is in accordance with the absorption spectrum in the visible region, as Figure 3 shows. Since the sec- ond harmonic of $1064 \mathrm{~nm}$ is still close to the long-wavelength tail of the absorption band, we can assume that also the $\chi^{(2)}$ parameter measured at this wavelength is affected by resonance enhancement to some extent. Although the value of $\chi^{(2)}$ is larger at $954 \mathrm{~nm}$, the noise level is higher because the pulse power fluctuations are increased by the nonlinear stimulated Raman process in the gas pressure cell.

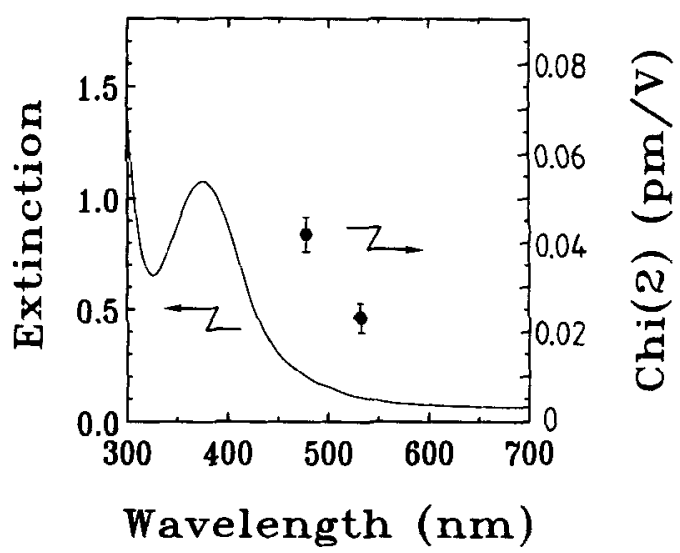

Fig. 3. Nonlinear susceptibilities $\chi_{33}^{(2)}$ of the data in Figure 2, plotted as a function of the generated second-harmonic wavelength (right scale). Also shown is the absorption spectrum of the sample in this wavelength region (left scale).

A very important property of $\chi^{(2)}$ materials is the temporal stability of the orientation of their chromophores. We measured the $\chi_{33}^{(2)}$ parameter of a sample containing the dye DR1 over a period of more than five months. The results are shown in Figure 4. The coefficient drops by a factor of

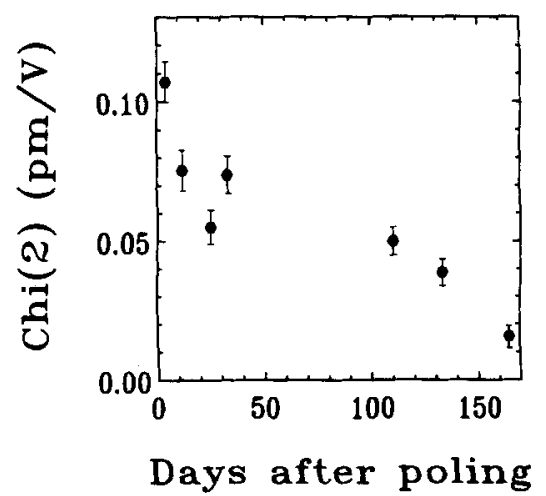

Fig. 4. Long-term behavior of the $\chi_{33}^{(2)}$ coefficient of a poled sample doped with the dye DR1.

roughly 3 (if the last data point is not taken into account) to 6 in this period. Thus, the stability is better than that of PMMA matrices doped with physically dissolved dye mole- 
cules (which have no chemical bond to the polymer backbone). ${ }^{[15]}$ We ascribe this effect to the rigidity of our three-dimensional crosslinked matrix material. However, the stability is not as good as in organic side-chain polymers. ${ }^{[16]}$ Also, the overall magnitude of the $\chi^{(2)}$ parameter is still rather small. Perhaps the electrical conductivity of the sample films in the early stages of the sol-gel process is still too high, so that the internal poling field strength and, as a consequence, the degree of orientation of the chromophores cannot reach high values. More work is needed to clarify this point.

The measurements presented in Figure 4 were carried out with infrared radiation of wavelength $1064 \mathrm{~nm}$. All the data points were obtained using the same values of the refractive indices $n_{\omega}$ and $n_{2 \omega}$. The scatter of the data may be due to the fact that spots of slightly different thickness were illuminated in the measurements. Note that the second harmonic generation signal varies with the square of the product of the $\chi^{(2)}$ parameter and sample thickness for very thin films. ${ }^{[13]}$ We wish to emphasize that these are first results and no optimization of the system parameters has been performed. For the side-chain chromophore ANB, the temporal stability of the $\chi^{(2)}$ parameter has not been investigated yet.

These first results show that the basic concept of using inorganic-organic composite materials produced by the solgel process as a rigid matrix for oriented $\chi^{(2)}$ chromophores is promising. The absolute $\chi^{(2)}$ values and the temporal stability of the DR1-doped samples are not satisfying at present, but no optimization has been carried out so far. Compared to conventional organic polymers such as PMMA doped with non-linked dyes, ${ }^{[15]}$ the decay is comparatively slow, indicating the effect of the three-dimensional crosslinked rigid matrix (although in new investigations of organic guest-host systems with higher glass transition temperatures considerably enhanced poling stabilities were found ${ }^{[17]}$ ). For the chemically linked chromophore (ANB), the temporal decay of the orientation has not yet been investigated but we anticipate similar improvements as known from organic side-chain chromophores. In further experiments, the matrix effect (composition, synthesis), the effect of dyes (structure, concentration) covalently linked to the inorganic backbone, and the influence of the processing parameters (variation of film thickness, poling conditions) will be investigated.

\section{Experimental}

As basic system, a composition based on $\mathrm{ZrO}_{2}$, methacrylic acid (MAA), and a methacryloxypropylsilane $\left(\mathrm{H}_{2} \mathrm{C}=\mathrm{C}\left(\mathrm{CH}_{3}\right) \mathrm{COO}\left(\mathrm{CH}_{2}\right)_{3} \mathrm{SiO}_{3 / 2}\right.$; MPTS) was used, as described in detail elsewhere ${ }^{[9,18]}$. The sols were synthesized by stepwise hydrolysis and condensation of the alkoxide precursors. Hydrolysis, condensation, $\mathrm{H}_{2} \mathrm{O}$ content, and silanol concentration were monitored by means of Karl-Fischer titration, IR, and ${ }^{29} \mathrm{Si}$ NMR spectroscopy. Homogeneous films of low optical loss could be synthesized by prehydrolyzing the silane with $0.5 \mathrm{H}_{2} \mathrm{O}$ per $-\mathrm{OR}$ and then adding $\mathrm{Zr}(\mathrm{OR})_{4}$ complexed with MAA $(\mathrm{Zr}: \mathrm{MAA}=1: 1)$. The chromophores were added in saturated tetrahydrofuran solutions $\quad(6.6 \mathrm{~g} / 1 \quad 4$-[2-(hydroxyethyl)- $N$-ethylamino]-4'-nitroazobenzene (DR1); 30 g/l 4-nitro-4'(oxypropyltrimethoxysilyl)azobenzene (ANB ${ }^{[19]}$ )).
After addition of a UV or thermal radical starter (1\% of 1-hydroxycyclohexylphenylketone or $1 \%$ of azoisobutyronitrile, respectively), the viscous sols were deposited by dip- or spin-coating on indiam-tin-oxide (ITO) coated glass slides. The samples were subjected to corona poling in a geometry with two thin wires positioned above the $1 \mathrm{TO}$ glass at a distance of about $15 \mathrm{~mm}$.

The breakdown voltage of the deposited films was measured according to the standard DIN 53481. During poling, the sol-gel process was initiated by UV irradiation and/or heating, depending on the starter substance. In the UV process, the sample was illuminated with the unfiltered radiation of a $200 \mathrm{~W} \mathrm{Hg}$ high-pressure lamp for 30 to $60 \mathrm{~s}$ and afterwards heated to $70^{\circ} \mathrm{C}$; in the purely thermal process the heating temperature was $120^{\circ} \mathrm{C}$. The samples were kept at the elevated temperature for about $1 \mathrm{~h}$. The thickness of the sample films was measured with a Sloan DEKTAK 3030ST stylometer.

The light source in our experimental set-up was a picosecond $\mathrm{Nd}^{2 \oplus)}$ : YAG laser (Continuum PY 601C-10) which provides pulses of 35 ps duration. Most measurements were performed directly with the $1064 \mathrm{~nm}$ output; however, a few experiments were also conducted with a second infrared wavelength $(954 \mathrm{~nm})$ which was generated in a Raman shifter (Spectra Physics RS-1) filled with pressurized $\mathrm{H}_{2}$ gas. The infrared radiation was split into two beams of equal power, which were focused onto the sample and on a reference material. The reference material was a poled PMMA layer with oxynitrostilbene side groups; it was held at a fixed angle and provided a reference signal to correct for laser power fluctuations. In both channels the generated second-harmonic light was separated from the fundamental wavelength by means of a monochromator (Jobin-Yvon H.10) and was detected by a cooled photomultiplier (Hamamatsu R649). The phototube signals were finally fed into a boxcar averager (Stanford Research SR245). A custom-built minicomputer controlled the experiment and the data acquisition. The signal was typically averaged over 60 laser shots at each orientation of the sample. The second-harmonic data were evaluated by fits of the corresponding theoretical functions [13]; the comparison with the amplitude of the fringe pattern of a $y$-cut crystalline quartz plate $\left(\chi_{11}^{(2)}=0.5 \mathrm{pm} /\right.$ $\mathrm{V}[20])$ yielded absolute values of the $\chi^{(2)}$ parameter. Note that all the $\chi^{(2)}$ values quoted in this paper correspond to the $d$ parameter in [13].

Received: November 27, 1992 Final version: January 29, 1993

[1] R. Dorn, D. Baums, P. Kersten, R. Regener, Adv. Mater. 1992, 4, 464.

[2] A. Buckley, Adv. Mater. 1992, 4, 153 .

[3] M. Eich, B. Reck, D. Y. Yoon, C. G. Willson, G. C. Bjorklund, J. Appt. Phys. 1989, 66, 3241.

[4] X. Zhu, Y. M. Chen, L. Li, R. J. Jeng, B. K. Mandal, J. Kumar, S. K. Tripathy, Opt. Commun. 1992, 88, 77.

[5] L. Yu, W. Chan, S. Dikshit, Z. Bao, Y. Shi, W. H. Steier, App. Phys. Lett. $1992,60,1655$.

[6] H. Schmidt, in Ultrastructure Processing of Advanced Materials (Eds: D. R. Uhlmann, D. R. Ulrich), Wiley, New York 1992, Ch. 38.

[7] H. Schmidt, in Chemical Processing of Advanced Materials (Eds: L. L. Hench, J. K. West), Wiley, New York 1992, p. 727.

[8] H. Schmidt, Mater. Res. Soc. Symp. Proc. 1992, 274, 121.

[9] H. Schmidt, H. Krug, R. Kasemann, N. Merl, V. Gerhard, F. Tiefensee, S. Brück, in Homage to Galileo (Ed: P. Mazzoldi), CLEUP, Padova 1992, p. 303 .

[10] J. Livage, C. Schmutz, P. Griesmar, P. Barboux, C. Sanchez, SPIE Proc. 1992, $1758,274$.

[11] H. Schmidt, H. Krug, R. Kasemann, F. Tiefensee, SPIE Proc. 1991, 1590, 36.

[12] P. D. Maker, R. W. Terhune, M. Nisenoff, C. M. Savage, Phys. Rev. Lett. 1962, 8, 21

[13] J. Jerphagnon, S. K. Kurtz, J. Appt. Phys. 1970, 41, 1667.

[14] P. N. Prasad, D. J. Williams, Introduction to Nonlinear Optical Effects in Molecules and Polymers, Wiley, New York 1991, p. 117.

[15] K. D. Singer, L. A. King, J. Appl. Phys. 1991, 70, 3251

[16] Y. Shi, W. H. Steier, M. Chen, L. Yu, L. R. Dalton, Appl. Phys. Lett, 1992, $60,2577$.

[17] M. Stähelin, D. M. Burland, M. Ebert, R. D. Miller, B. A. Smith, R. J. Twieg, W. Volksen, C. A. Walsh, Appl. Phys. Lett. 1992, 6t, 1626.

[18] R. NaB, H. Schmidt, E. Arpac, SPIE Proc. 1990, 1328, 258.

[19] H. Dürr et al., unpublished results.

[20] S. K. Kurtz, J. Jerphagnon, M. M. Choy, in Landolt-Börnstein Numerical Data and Functional Relationships in Science and Technology, New Series, Group III : Crystal and Solid State Physics, Vol. 11, Springer, Berlin 1979, p. 671 . 